\title{
PHIPS-HALO: the airborne Particle Habit Imaging and Polar Scattering probe - Part 3: Single-particle phase discrimination and particle size distribution based on the angular-scattering function
}

Fritz Waitz et al.

Correspondence to: Fritz Waitz (fritz.waitz@kit.edu) and Emma Järvinen (emma.jaervinen@kit.edu)

The copyright of individual parts of the supplement might differ from the article licence. 


\section{S1 - Calibration Data set for the Phase Discrimination Algorithm and Particle Sizing}

The calibration of the ice discrimination algorithm as well as for the particle sizing are based on manual classified particles from the ACLOUD and SOCRATES campaigns as introduced in section 2. An overview of the diameter of the particles in those two data sets is shown in Fig. S1. An overview of the different habits observed during the two campaigns in different size

5 regimes is shown in Fig. S2 and Fig. S3. Note that during the first flight of SOCRATES, PHIPS was not recording images due to a laser malfunction, hence RF01 is excluded from the calibration and analysis. RF15 was dominated by precipitation (such as seen in Fig. S8) and was hence excluded as well, as those particles are not representative (see discussion in section ) and would negatively affect the calibration.
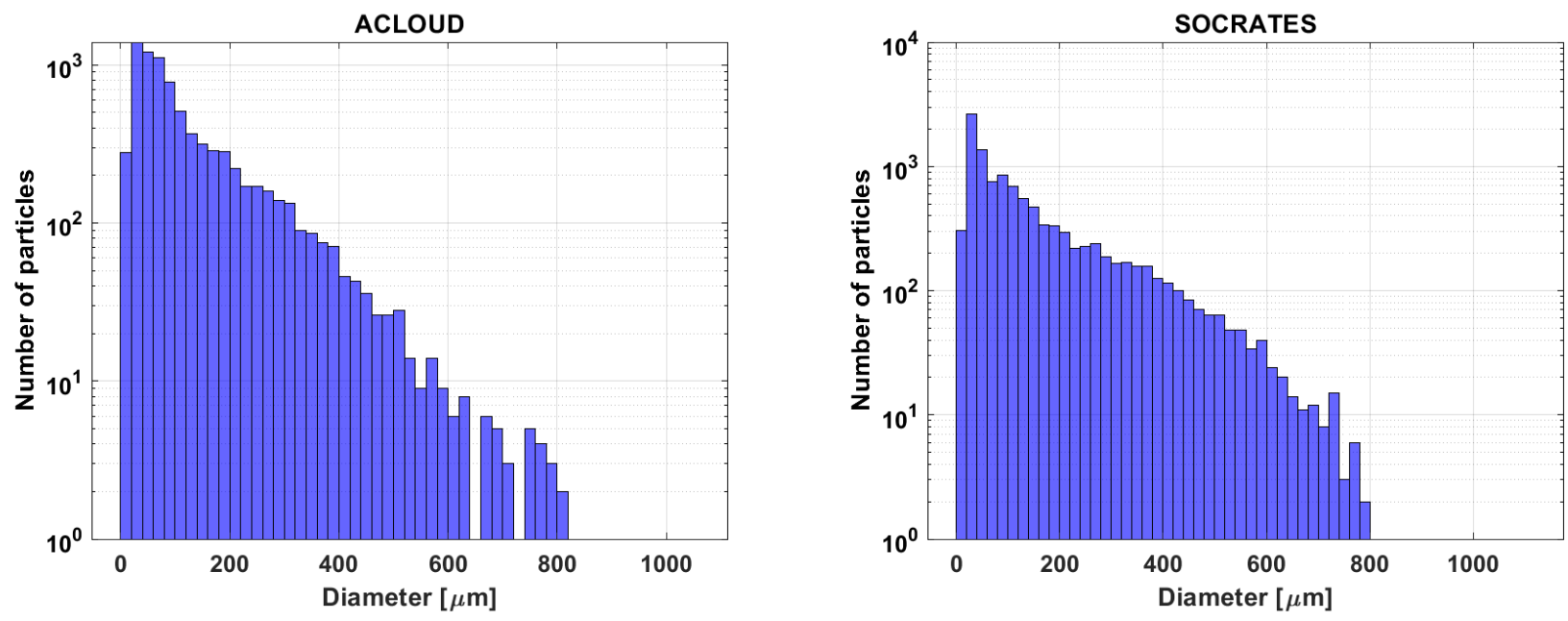

Figure S1. Overview of the diameter of the manual classified particles of ACLOUD (left) and SOCRATES (right).

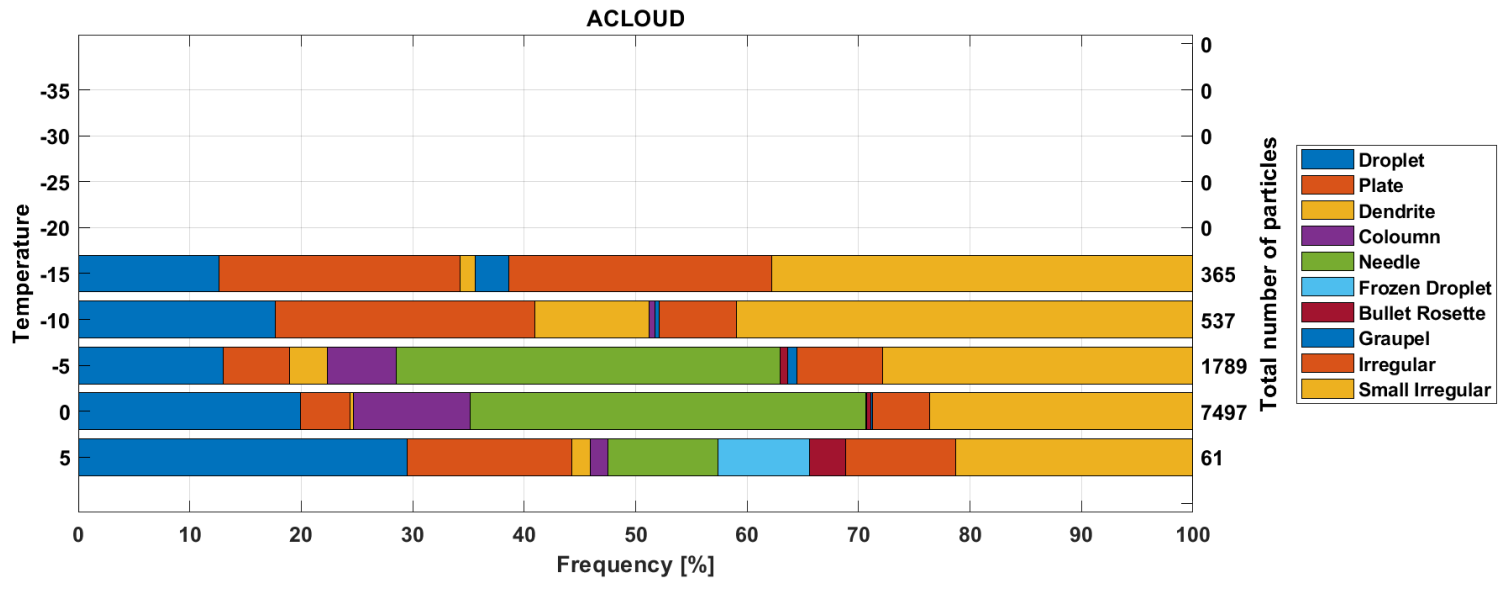

Figure S2. Overview of the shape of the manual classified particles of ACLOUD in different temperature regimes. 


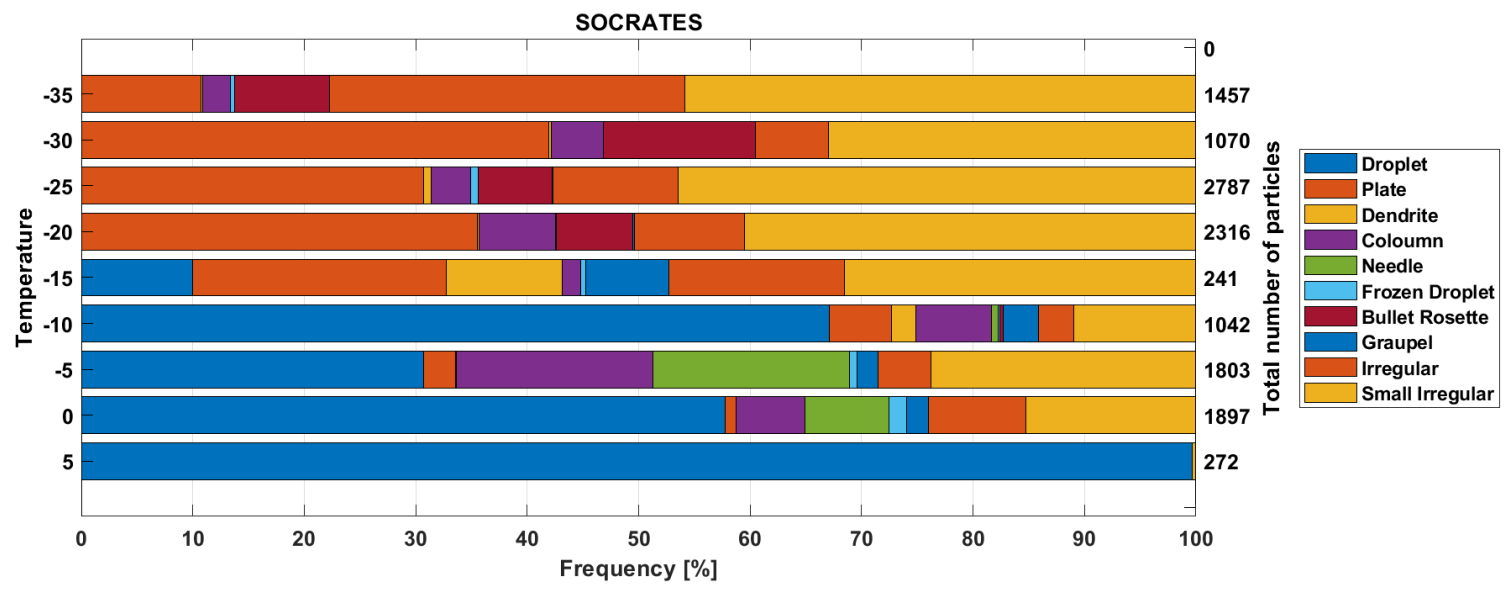

Figure S3. Overview of the shape of the manual classified particles of SOCRATES in different temperature regimes.

\section{S2 - $f_{1}$ : Mie Feature}

10 As explained in Sec. 3, the idea behind the first discrimination feature $f_{1}$ is to compare a particles ASF with the calculated Mie ASF for a spherical particle. Now the problem is, that the Mie ASF is dependent on the particle diameter, which is unknown. In theory, it could be possible to calculate the particle diameter based on its ASF as explained in Sec. 4. However, this is again dependent on the particle's phase, which is again unknown. Furthermore, as the Mie calculation is rather time consuming $(\approx 10-20$ s per particle), it would significantly increase computation time for the phase discrimination (otherwise $<1$ s per 15 particle).

Due to the coarse angular resolution however, the Mie scattering intensity of a spherical particle is approximately proportional to its diameter squared and thus $I_{\mathrm{Mie}}(\theta, d) / D^{2} \simeq$ const., as shown in Fig. S4. Hence, the ratio $q$ is $\simeq$ const. and $q / \bar{q}$ is almost independent of the diameter used for the Mie calculation. Therefore, the reference Mie ASF used for the comparison feature $f_{1}$ is based on a particle of fixed diameter $D=100 \mu \mathrm{m}$.
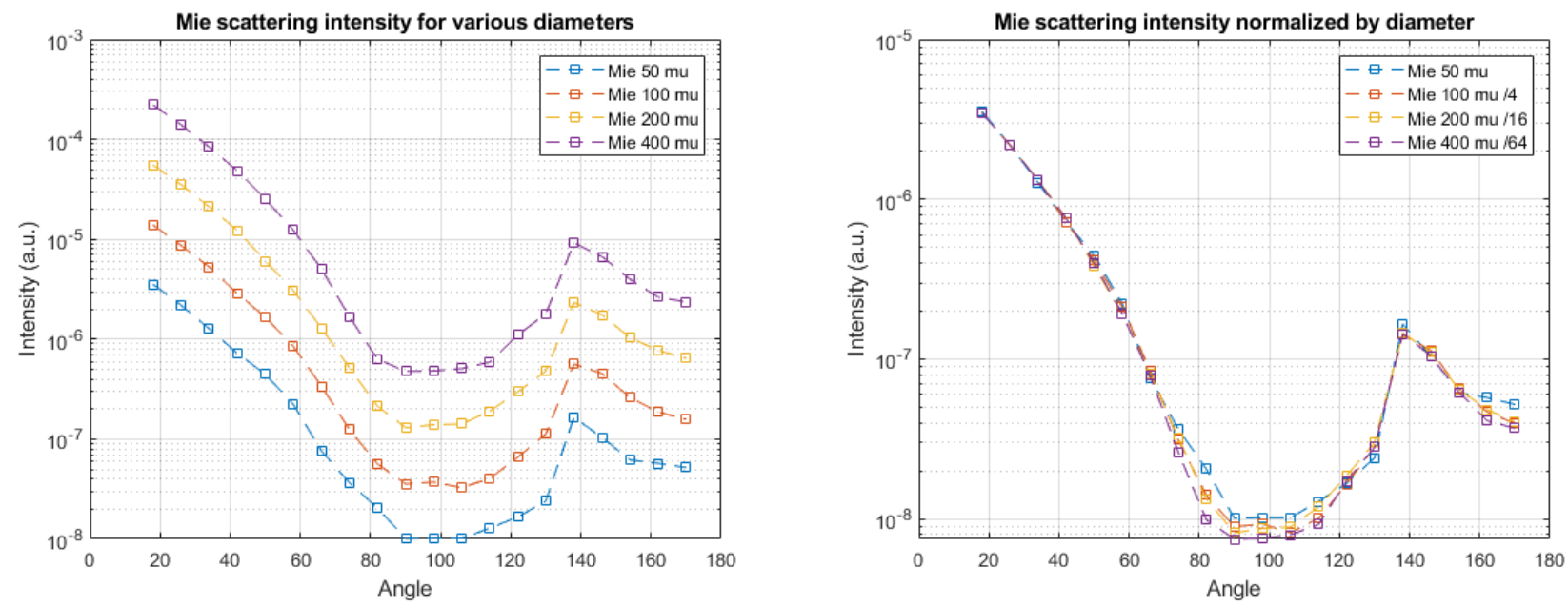

Figure S4. Calculated Mie intensity for droplets of various size (left) and the same graph normalized by diameter (right). 
In theory, a third slope feature after the rainbow peak could be calculated from the maximum in said range to the end. However, in comparison to the other features, the histogram of the potential third slope feature would show a much bigger overlap (see Fig. S5). Possible explanations for this could be the limited amount of available channels in the backwards direction or shifting of the rainbow peak due to deformation of elongated droplets (more on that in section S4). Further, depending on particle orientation, specular reflections on pristine ice particles can lead to large peaks in the ASF. Such a peak could erroneously "trigger" this "rainbow feature. Hence, since the inclusion of this feature could not increase the phase discrimination accuracy, it is not used further as a classification feature.

To compensate the impact of specular reflections (i.e. that those peaks are falsely identified as very steep slopes and ice particles are misclassified as droplets), it was considered to look at the slope of every individual channel, i.e. the ratio to the next channel

$m_{i}=\log \left(\frac{I\left(\theta_{i+1}\right)}{I\left(\theta_{i}\right)}\right)$.

As already utilised for the down slope $\left(f_{2}\right)$ feature, according to Mie theory, for droplets, the scattering intensity descends continuously for increasing $\theta$ until the minimum around $90^{\circ}$. Hence, if the scattering intensity suddenly increases during the down slope, this could be caused by a specular reflection peak. Analogously, the same goes for peaks during the up slope $\left(f_{3}\right)$.

However, for small particles with scattering signal near the magnitude of the background of only a couple counts, noise can falsely be interpreted as a specular reflection peak. Also, similar to the down slope after the rainbow, the histogram of the feature distribution also show large overlaps and implementing one or more specular reflection peak features could not improve the discrimination accuracy. Hence, in an effort to keep the discrimination algorithm as simple as possible and the number of discrimination features low, specular reflection peaks are not taken into account.

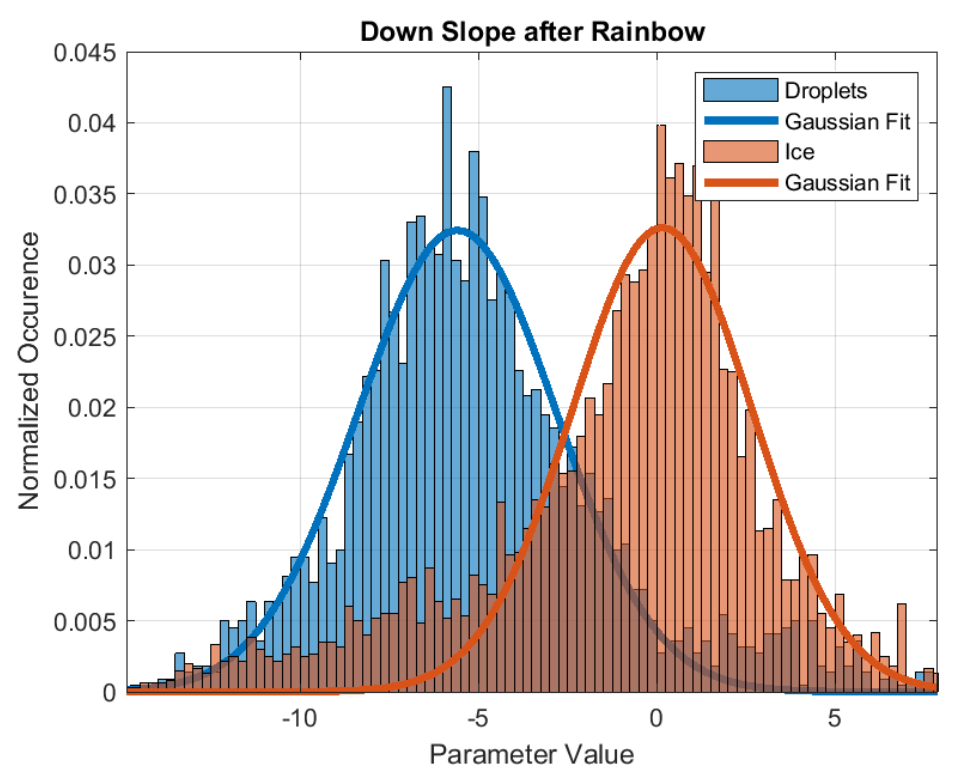

Figure S5. Distribution of the feature parameter for the down slope after rainbow peak, based on the manual classified images from the SOCRATES campaign. 
The phase discrimination algorithm gives not only a binary classification result (e.g. $0=$ ice, $1=$ droplet) but also a discrimination confidence. Fig. S6a shows, that correctly classified particles are usually classified with high confidence (close to 0 or $100 \%$ ), whereas misclassified particles are often times quite unclear and the classification confidence is near 50\%, i.e. the different features are equally strong leaning towards both sides. This can be used to manually check the ASF (or image, wherever available) of individual particles, where the algorithm "is not sure".

a)

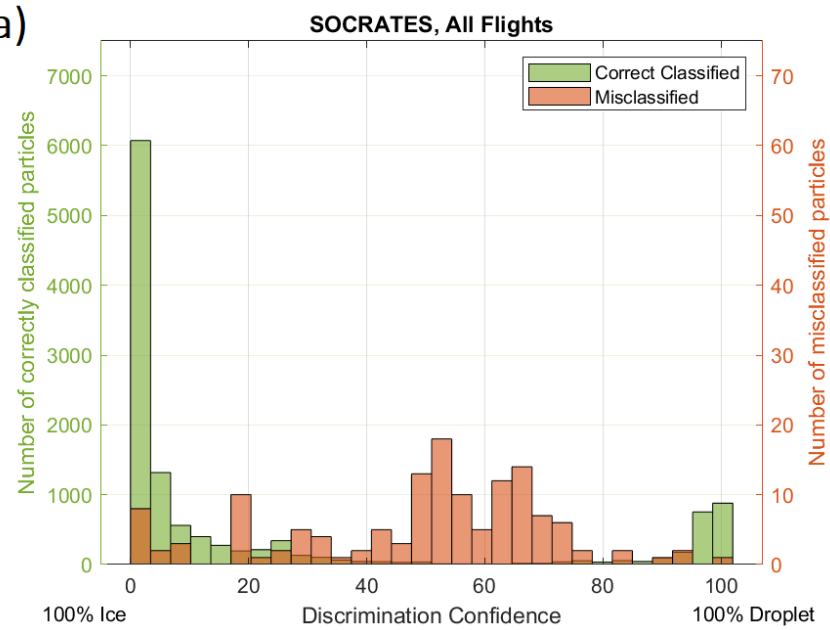

b)

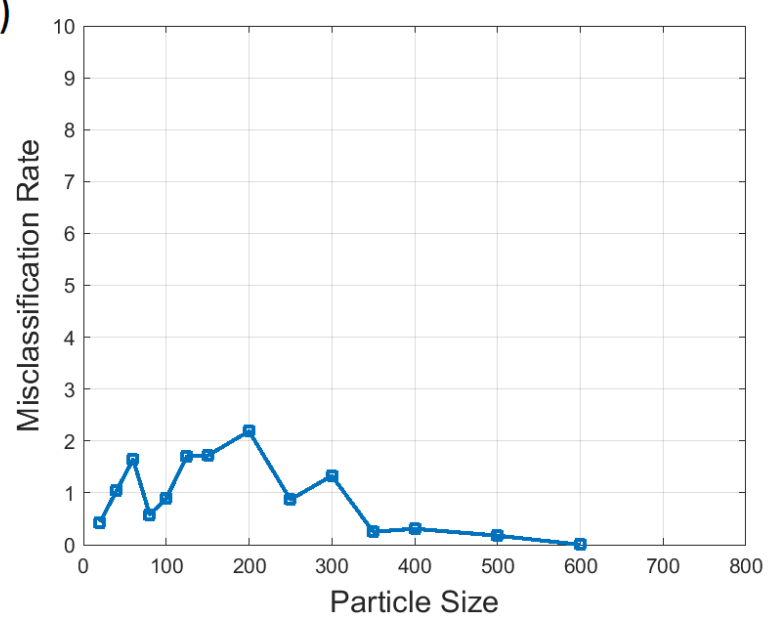

Figure S6. a) Discrimination confidence of all correctly classified (left) and misclassified particles (right). b) Correlation of discrimination accuracy and particle size. The size binning is the same as used previously.

In Tab. 1, the discrimination accuracy for the various different particle habits for the SOCRATES data set is shown. It can be seen, that the discrimination accuracy is relatively constant for the different habits and the algorithm is not biased towards certain shapes. Also, no correlation of discrimination accuracy and particle size was found (see Fig. S6b.). In this section, examples of typical particle types that are sometimes misclassified, are presented.

Table 1. Discrimination accuracy for different particle habits for the SOCRATES data set.

\begin{tabular}{l|lllllll} 
Particle Shape & Droplets (all) & Elongated Droplet & Drizzle & Ice Particles (all) & Column & Plate & Needle \\
\hline Number True & 2189 & 267 & 13 & 9907 & 788 & 2456 & 423 \\
Number False & 95 & 40 & 6 & 29 & 6 & 2 & 2 \\
Accuracy [\%] & 95.8 & 87 & 68.4 & 99.7 & 99.2 & 99.9 & 100 \\
\hline \hline Particle Shape & Bullet Rosette & Graupel & Frozen Droplet & Irregular & Pristine & Rimed & Aggregate \\
\hline Number True & 618 & 127 & 56 & 5339 & 136 & 640 & 724 \\
Number False & 0 & 0 & 1 & 20 & 2 & 1 & 2 \\
Accuracy [\%] & 99.9 & 100 & 98.2 & 99.6 & 98.6 & 99.8 & 99.7
\end{tabular}

\section{Elongated Droplets}

The largest challenge for the presented discrimination algorithm is the correct classification of "elongated" droplets as seen in Fig.S7. Those are droplets that are deformed due to the pressure difference in the inlet of the probe. This affects about $12 \%$ of all droplets. Due to this deformation, the droplets are no longer spherical and harder for the algorithm to classify 
correctly. Whereas droplets usually show a steep decline in scattering intensity towards the side (i.e. the minimum around $90^{\circ}$ ), elongated droplets tend to scatter more light in the sideways direction. Also, the rainbow peak can be shifted. Still, despite their asphericity, the algorithm can still detect some features and about $87 \%$ of all elongated droplets can be classified correctly.
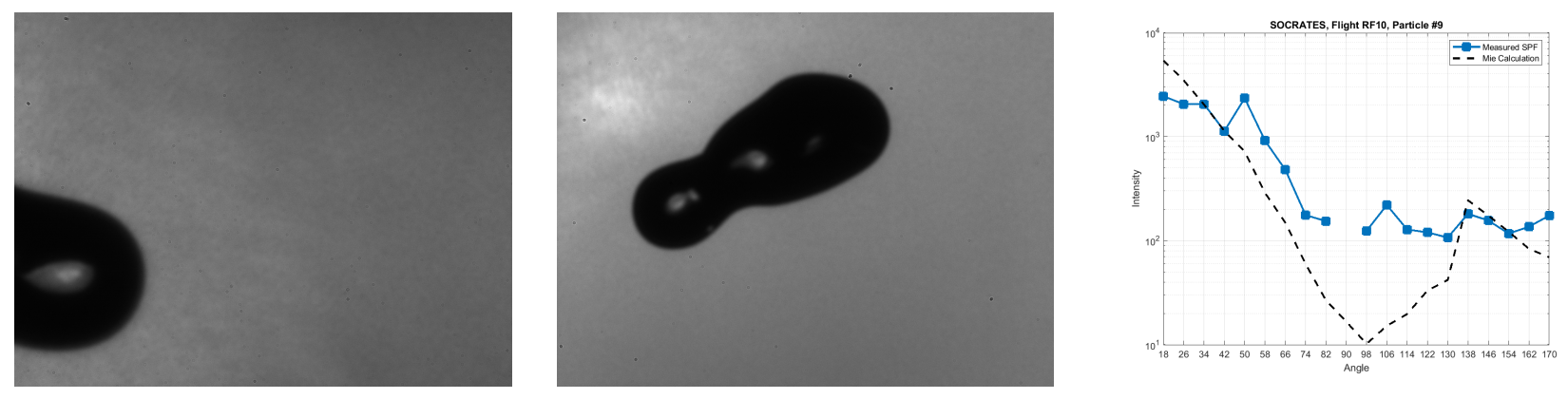

Figure S7. Example of a typical elongated droplet, stereo images (left: CTA1, middle: CTA2) and the corresponding ASF (right) including the theoretical Mie ASF of a spherical particle $(D=200 \mu m)$ for comparison.

\section{Large Drizzle Droplets}

Another difficult case is the classification of very large particles which cause a lot of the scattering channels to be saturated. Since saturated channels are excluded from the analysis, the slope features cannot be calculated for such particles. Since the integrated area between the ASF and Mie calculation (see Mie feature $f_{1}$ ) is very large (see ASF in Fig.S8), such particles are usually classified as ice. Generally, this is reasonable, since large particles $>500 \mu m$ are usually ice particles. However, deformed precipitating drizzle droplets (see Fig.S8) can be misclassified. Such cases are, however, very rare.

A similar issue exists for very small particles with ASF near the background. If a particle shows an equally low scattering intensity over all channels, the resulting slopes are relatively horizontal and the integral between the Mie calculation large. The particle is hence usually classified as ice. This, however, is not a problem since PHIPS does not trigger on droplets below $50 \mu m$ and all smaller particles can thus be assumed to be ice.
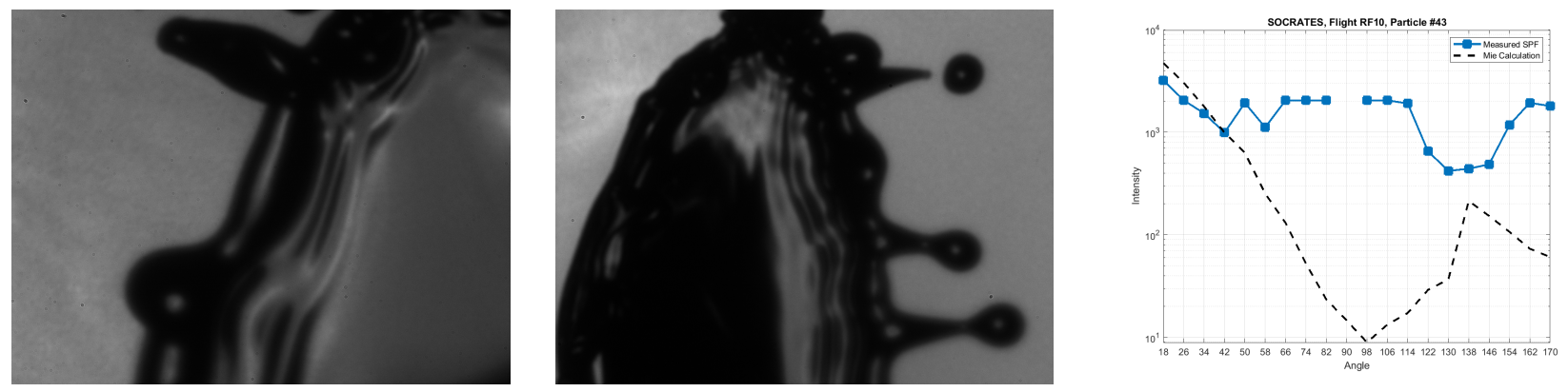

Figure S8. Example of a typical misclassified large drizzle droplet with multiple saturated channels.

\section{Other Misclassified Particles}

Fig.S9 shows an example of a particle that is clearly a pristine ice crystal, which is misclassified because the ASF resembles all typical features of a droplet's: the minimum around $90^{\circ}$, the steep slopes. There is even a specular reflection in the angular range of the rainbow peak. Note that the lower ASF in the first scattering angles compared to the theoretical ASF does not influence the classification result, since the first channels are not taken into account as they are often times saturated. Approaches that, for example, only exclude the first channels if they are saturated and include them otherwise, were tried and could be able 
to correctly classify particles as this one, but resulted in an overall decrease of the discrimination accuracy (i.e. particles that are classified correctly now would then be misclassified). The algorithm was calibrated to optimize the overall discrimination accuracy, i.e. that the highest fraction of particles is classified correctly. Hence, if a particle's ASF happens to look like this, it will be misclassified. Fortunately, such cases are very rare.
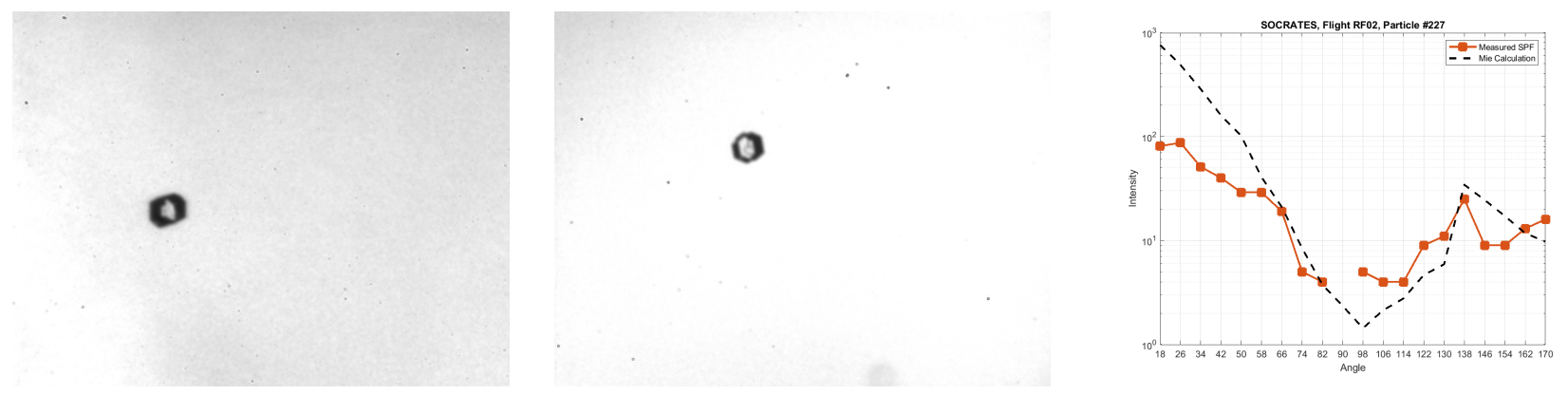

Figure S9. Example of a particle that is clearly a pristine ice crystal that is misclassified because the ASF resembles all typical features of a droplet's.

\section{S5 - Examples of Shattering Cases}

In Fig.S10, a typical example of a shattering event is shown. Fig.S11 shows the microscopic stereo images of a case where a large aggregate was broken up into multiple small fragments.
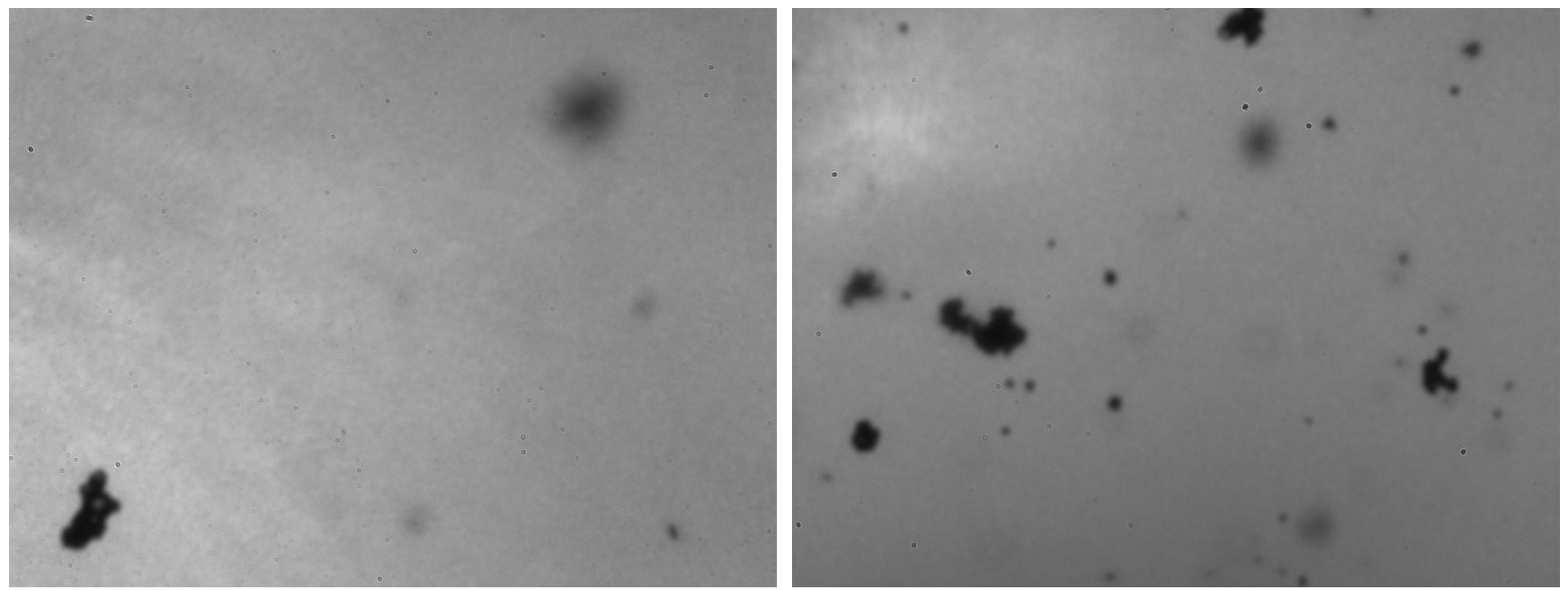

Figure S10. Example of a shattering event during the transit leg of SOCRATES RF06. The images (left CTA1, right CTA2) show one representative shattering event during this time period. 

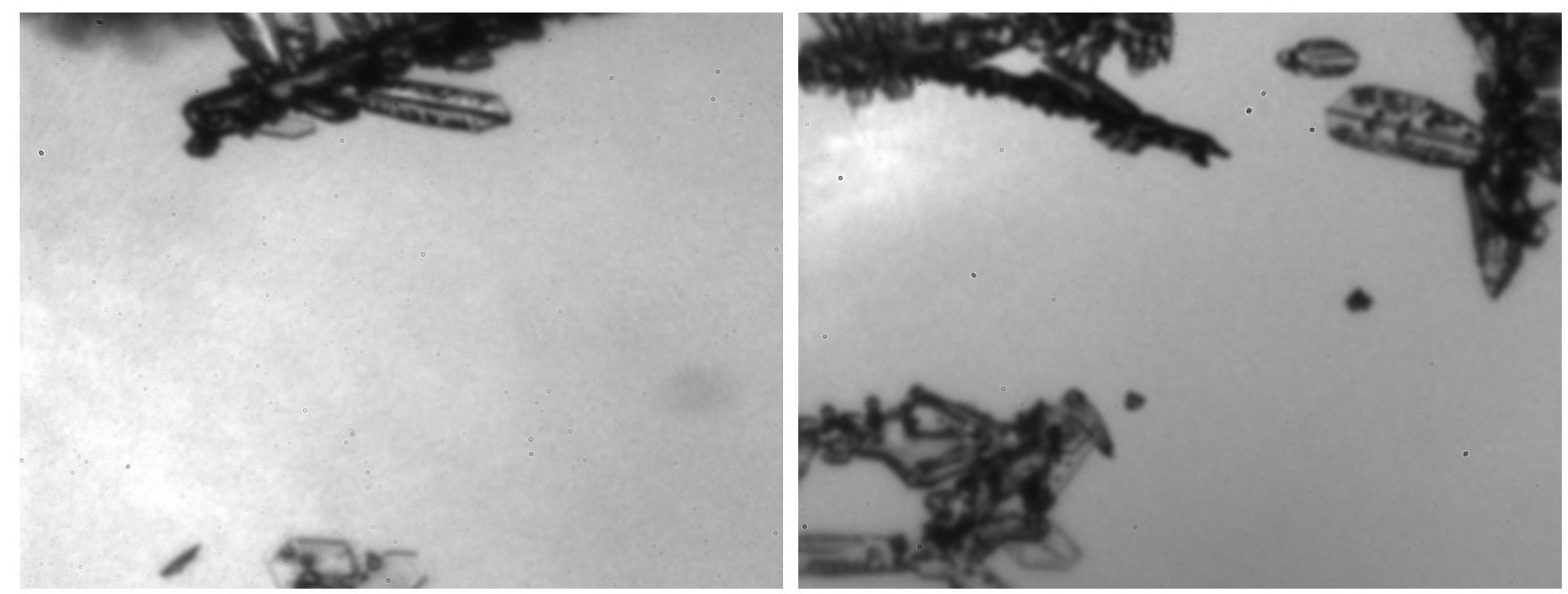

Figure S11. Example of a shattering event (left CTA1, right CTA2) during another segment of RF06, indicating the breaking up of aggregates.

\section{S6 - Integration of theoretical ASF over Nephelometer Field of View}

The polar nephelometer part of PHIPS consists of 20 parabolic mirrors (channels) that collect the scattered light before it is guided via PMMA fibers to the photomultiplier. The total amount of measured light per channel $I_{\text {channel }}$ is hence the integral over the surface of the detector $A$, i.e. the total intensity that arrives in the solid angle $\Omega$ of each parabolic mirror

$$
I_{\text {channel }}=\iint_{A} I(\Omega) d \Omega
$$

85 Due to the azimuth symmetry of the detectors, the double integral can be analytically solved with respect to the azimuthal angle, and the resulting integral only depends on the polar angle, $\theta$, as follows

$$
I=2 \pi r^{2} \int_{\theta_{0}-\alpha}^{\theta_{0}+\alpha} I(\theta) \cdot \arctan \left(\sqrt{\tan ^{2}(\alpha)-\tan ^{2}\left(\theta-\theta_{0}\right)}\right) d \theta
$$

where $r$ is the distance from the scattering center to the detector $(r=83 \mathrm{~mm}$, see Abdelmonem et al. (2016)), $\alpha$ is the half opening angle $\alpha=\arctan \left(\frac{R}{r}\right)=3.5^{\circ}$ of the detector with the radius $R=5 \mathrm{~mm}$ and $\theta_{0}$ marks the center of the detector.

Figure S12 shows an example of this integration in a case of spherical particle. The ASF of a theoretical spherical particle with $\mathrm{D}=200 \mu \mathrm{m}$ calculated using the Mie theory is shown in blue. The red curve shows the same ASF integrated over the field of view of the 20 nephelometer channels. 


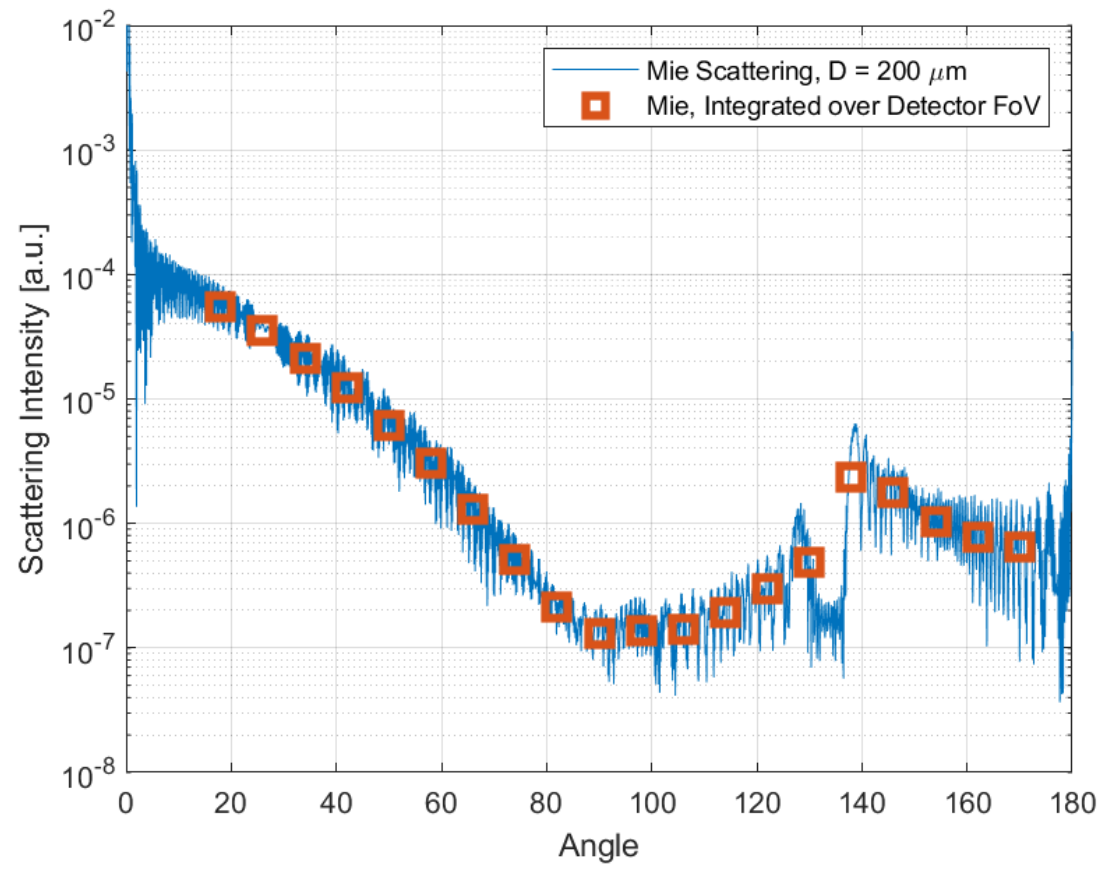

Figure S12. Calculated ASF of a droplet with $D=200 \mu \mathrm{m}$ (blue) and the same ASF integrated over the field of view of the nephelometer channels (red). 